\title{
EL COMPROMISO DE CONTINUIDAD Y EL VÍNCULO DEL CONSUMIDOR CON LA ORGANIZACIÓN"
}

\author{
Ana María Arboleda Arango**
}

* doi: 10.11144/Javeriana.cao29-53.ccvc. Este artículo es producto de la investigación “El contrato psicológico del consumidor", financiado por la Universidad Icesi, desde enero de 2010 hasta noviembre de 2013. El artículo se recibió 07-11-2013 y se aprobó el 10-10-2016. Sugerencia de citación: Arboleda A., A. M. (2016). El compromiso de continuidad y el vínculo del consumidor con la organización. Cuadernos de Administración, 29 (53), 181-201. http://dx.doi.org/10.11144/Javeriana.cao29-53.ccvc.

** PhD en Administración de la Universidad Tulane, Nueva Orleans (LA), Estados Unidos, 2011. Profesora de tiempo completo del Departamento de Mercadeo y Negocios Internacionales de la Universidad Icesi, Cali, Colombia.

Correo electrónico: amarboleda@icesi.edu.co 


\section{El compromiso de continuidad y el vínculo del consumidor con la organización}

\section{Continuance commitment \\ and consumer's bond with the organization}

\section{ResUMEN}

Este artículo evalúa en qué medida el compromiso de continuidad del consumidor hacia una organización modera la relación entre su compromiso afectivo e intención conductual. Se operacionaliza el constructo compromiso de continuidad de dos formas: la posibilidad de un consumidor para elegir el prestador de servicios de salud y el costo de cambiarse de institución educativa al tener una beca. Los resultados muestran que el compromiso afectivo incrementa la intención conductual hacia la organización. Esta relación decrece cuando la continuidad se refiere al costo y cuando se refiere a la posibilidad de elegir el proveedor del servicio. Futuros estudios podrían replicar esta metodología para el caso de marcas o productos de consumo masivo, y de otro lado, podrían confirmar la experiencia emocional de los consumidores que reciben beneficios adicionales.

Palabras clave: comportamiento del consumidor compromiso afectivo, compromiso de continuidad.

Clasificación JEL: $\mathrm{M}_{3} \mathrm{O}, \mathrm{M}_{3}$

\section{ABSTRACT}

This article evaluates the extent to which a consumer's continuance commitment to an organization moderates the relationship between his affective commitment and behavioral intention. The continuance commitment is operationalized in two ways: the possibility a consumer has to choose a healthcare organization and the cost of switching from an education institution by having a scholarship. Results show that the affective commitment increases behavioral intentions towards the organization. This relationship decreases when the continuance commitment relates with the cost and the possibility of choosing the service provider. Future studies could replicate this methodology for cases dealing with brands or massive consumption products and, on another side, could confirm the emotional experience of those consumers who receive additional benefits.

Keywords: Consumer behavior, affective commitment, continuance commitment.

JEL classification: $\mathrm{M}_{3} \mathrm{O}, \mathrm{M}_{3} 1$

\section{0 compromisso de} continuidade e o vínculo do consumidor com a organização

\section{RESUMO}

Este artigo avalia em que medida o compromisso de continuidade do consumidor com uma organização modera a relação entre seu compromisso afetivo e intenção comportamental. Operacionaliza-se o constructo compromisso de continuidade de duas formas: a possibilidade de um consumidor para escolher o prestador de serviços de saúde e o custo de mudar-se de instituição educativa ao ter uma bolsa. Os resultados mostram que o compromisso afetivo aumenta a intenção comportamental com a organização. Esta relação decresce quando a continuidade se refere ao custo e quando se refere à possibilidade de escolher o prestador do serviço. Futuros estudos poderiam aplicar esta metodologia para o caso de marcas ou produtos de consumo em massa, e por outro lado, poderiam confirmar a experiência emocional dos consumidores que recebem benefícios adicionais.

Palavras-chave: comportamento do consumidor, compromisso afetivo, compromisso de continuidade.

Classificação JEL: $\mathrm{M}_{3} \mathrm{O}, \mathrm{M}_{3}$ 


\section{Introducción}

El compromiso afectivo es una ventaja competitiva para una organización cuando un consumidor considera un producto o servicio como único entre los demás de la categoría por el significado emocional que tiene para él (Hur, Park y Kim, 2010). Estudios previos muestran que el compromiso afectivo del consumidor conlleva a una conducta positiva en términos de recompra o intención de continuar usando la marca, pero este resultado cambia si el consumidor siente que está limitado a permanecer en la organización (Fullerton, 2003, 2005). Esta restricción se conoce como compromiso de continuidad, el cual se refiere a la escases de alternativas de cambio y a los costos asociados al cambio (Allen y Meyer, 1990; Fullerton, 2003).

El propósito de este artículo es determinar en qué medida el compromiso de continuidad que debe asumir el consumidor con una organización modera la relación entre su compromiso afectivo e intención conductual. Se utiliza el constructo compromiso afectivo como variable independiente y antecedente de la intención conductual del consumidor; y se utilizan dos definiciones del constructo compromiso de continuidad como variable moderadora. Existe una tercera forma de compromiso hacia una organización que no se estudia en el artículo: el compromiso normativo que se refiere a la percepción de la obligación que percibe el individuo hacia la organización como una retribución a lo que esta le ofrece o le ha dado (Allen y Meyer, 1990).

La literatura en compromiso organizacional define el compromiso de continuidad como la dificultad que siente el consumidor para cambiar la relación con una organización porque la oferta alternativa es limitada o por el costo asociado al cambio (Fullerton, 2005). Consistente con la literatura, este artículo entiende el compromiso de continuidad hacia una organización a través de dos eventos que restringen al consumidor de realizar un cambio de dicha organización por otro oferente. Cada uno de estos eventos se trata de manera independiente. El primer evento se refiere a una restricción en la posibilidad de elegir sustitutos en el mercado (estudio 1 ) y el segundo, al costo asociado a la pérdida de continuidad (estudio 2). Las dos formas en que estos estudios operacionalizan el constructo compromiso de continuidad son complementarias entre sí porque evalúan la dependencia en una organización a través de dos condiciones opuestas. El primer estudio utiliza directamente una limitación, mientras que el segundo estudio utiliza un beneficio.

Adicionalmente, la operacionalización del compromiso de continuidad que ofrece el presente artículo difiere de la forma tradicional, la cual utiliza una escala Likert en la 
que el sujeto indica su percepción de continuidad con la organización (Fullerton, 2005). Este estudio hace una definición más simple y objetiva: el hecho de poder cambiar de prestador de servicios de salud y el hecho de tener una beca educativa. En cambio, tradicionalmente el compromiso de continuidad se evalúa teniendo en cuenta la percepción del costo asociado al cambio y de la dificultad que tiene el consumidor para realizar un cambio, pero no se operacionaliza como la posibilidad de cambio teniendo en cuenta la oferta del mercado. Esta forma de medición es coherente con la definición del constructo.

Así mismo, esta operacionalización del constructo fortalece la investigación en términos de validez externa mostrando, primero, que es posible definir el concepto de dos formas diferentes y complementarias y, segundo, que existen alternativas más concretas, a la evaluación subjetiva del consumidor, que responden a la definición conceptual del constructo.

Los resultados muestran que tener la opción de elegir el proveedor del servicio y tener beneficios asociados al costo se relaciona con un aumento en la intención conductual positiva hacia la organización. Sin embargo, esto no necesariamente cambiará su sentimiento de vínculo hacia la organización a menos que implique un costo.

De manera práctica, los resultados de estos dos casos soportan la idea de pensar cuidadosamente las estrategias relacionales o de Consumer Relationship Marketing para vincular al consumidor con la organización alertando sobre las consecuencias de que estas estrategias sean percibidas por el consumidor como una obligación.

\section{El Compromiso Afectivo del Consumidor}

El compromiso afectivo es una conexión emocional que un individuo experimenta hacia una organización. Esta conexión denota que existe un vínculo emocional, una identificación e involucramiento con una organización (Meyer, Stanley, Herscovitch y Topolnytsky, 2002). El compromiso afectivo es un constructo inicialmente elaborado para evaluar el vínculo entre un empleado y una organización, sin embargo el concepto aplica para la relación consumidor-organización y además ha sido utilizado en el mercadeo relacional (Fullerton, 2005).

El compromiso afectivo es para la teoría del consumidor un componente psicológico a través del cual el consumidor establece un vínculo con una organización, con una marca o incluso con los empleados como representantes de la organización (Jones, Fox, Taylor 
y Fabrigar, 2010). Adicionalmente, la idea de vínculo se remite a la teoría del vínculo emocional bajo la cual se argumenta que el consumidor busca la proximidad a otra persona (e.g., proveedor de servicio) u objeto hacia el cual siente un compromiso afectivo (Evanschitzky, Brock y Blut, 2011). El consumidor desarrolla un compromiso afectivo en la medida en que siente que la organización o la marca le brindan un espacio no solo físico sino emocional (Bowlby, 1977). Es en este espacio psicológico en el que el consumidor se siente seguro para experimentar y tomar decisiones de consumo, por ejemplo decidir utilizar un producto o servicio.

Consumidores que piensan que la organización provee lo que ha prometido tienden a sentir que esta es una entidad confiable y sienten un compromiso más fuerte (Morgan y Hunt, 1994). El compromiso afectivo se relaciona con la idea de que la organización está interesada en el bienestar del consumidor y no llevará a cabo acciones que tengan un impacto negativo sobre él (Wetzels, de Ruyter y van Birgelen, 1998). Consecuentemente, el compromiso de tipo afectivo está relacionado con intenciones conductuales positivas como el continuar como clientes de una organización en el futuro (Huang, Cheng y Farn, 2007; Hur et al. , 2010; Jones et al., 2010; Wetzels et al. , 1998). Bajo este marco conceptual se propone la siguiente hipótesis:

Hipótesis 1: El compromiso afectivo del consumidor hacia una organización se relaciona positivamente con su intención conductual hacia la misma.

\section{La posibilidad de elegir: restricción en la oferta de servicios de salud}

La restricción en la posibilidad de elección puede generar la percepción de obligación a permanecer con la institución. Esta definición es coherente con la idea de compromiso de continuidad (Fullerton, 2003, 2005). En general, un individuo deseará tener primero la posibilidad de elegir y segundo contar con un número razonable de opciones a partir de las cuales realizar su elección (Bettman, Luce y Payne, 1998; Chernev, 2011). Adicionalmente, cuando un consumidor tiene claro el producto o servicio que requiere preferirá elegir entre una oferta relativamente amplia (Chernev, Mick y Johnson, 2003). Categorías de productos o servicios con una oferta restringida pueden asociarse con mercados poco competitivos en el que no haya un esfuerzo por parte de las marcas por mejorar su calidad (Gautam y Kumar, 2011; Zhao, Zhao y Helsen, 2011). Incluso, las estrategias organizacionales que generan la percepción de monopolio o de que solo existen pocos oferentes generan en el consumidor sentimientos de desconfianza (Sundie, Gelb y Bush, 2008). 
Este primer estudio tiene el objetivo de determinar en qué medida el compromiso de continuidad de un consumidor de servicios de salud modera la relación entre su compromiso afectivo e intención conductual hacia una institución. El compromiso de continuidad no es una decisión del consumidor, es una condición que está dada por el entorno y en este caso específicamente por el carácter competitivo del sector. La competitividad se refiere a un entorno en el que las empresas realizan cambios para atraer a los consumidores, estos cambios pueden estar asociados al precio o la calidad del servicio. La implementación de estrategias competitivas motiva al consumidor al cambio buscando el mejor oferente de acuerdo con sus necesidades.

En este sentido el compromiso de continuidad implica que el consumidor no puede elegir o cambiar el prestador de servicios de salud. Esta restricción se puede explicar de dos formas, por la oferta de servicios de salud en el mercado y por el plan de salud con el que cuenta un consumidor. La oferta de servicios de salud es el tamaño o el número de instituciones de salud disponibles para el consumidor en su área geográfica de acuerdo con las necesidades del servicio. La segunda condición que restringe la elección es el plan de salud al que un individuo está vinculado. En algunos casos el plan de salud obliga a recibir los servicios en una institución en particular, limitando la posibilidad de elegir un prestador de servicios diferente entre cualquiera de las instituciones disponibles.

De esta manera, en el caso en el que el consumidor tiene la posibilidad de elegir su institución de salud de acuerdo con las características de la oferta, se puede fortalecer la relación positiva entre su compromiso afectivo e intención conductual hacia la institución. En el caso contrario, cuando el consumidor no tiene la posibilidad de elegir, un aumento en el compromiso afectivo disminuiría su intención de regresar a la institución (figura 1). Esta relación inversa se puede explicar debido a que la comparación no es posible para el consumidor. Los consumidores que tienen la posibilidad de comparar y evaluar la opción de cambio están más convencidos de su elección y son menos propensos al cambio (Chernev et al., 2003). Consecuentemente se proponen la siguiente hipótesis:

Hipótesis 2: La relación entre el compromiso afectivo de un consumidor y su intención conductual hacia una institución es más fuerte cuando las personas tienen la posibilidad de elegir que cuando no tienen esta posibilidad. 


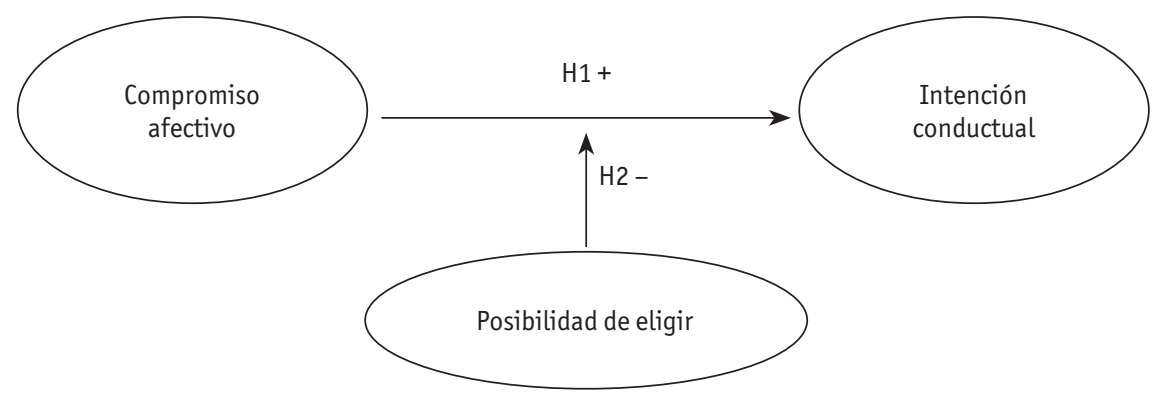

Figura 1. Compromiso de continuidad: la posibilidad de elegir

Fuente: elaboración propia.

\subsection{Estudio 1}

A través de un primer estudio se evalúo de manera transversal, primero, la relación entre el compromiso afectivo y la intención conductual y, segundo, el efecto moderador del compromiso de continuidad con una institución prestadora de servicios de salud. Se encuestaron 473 personas que habían visitado como paciente una institución prestadora de servicios de salud en el último mes. Los sujetos fueron estudiantes universitarios quienes decidieron voluntariamente participar en el estudio. Después de eliminar las encuestas no válidas el tamaño de la muestra fue de 466. En la muestra final, el $60 \%$ fueron mujeres y el 40\% fueron hombres, con una edad promedio de 22,3 años (desv.st $4,89)$. Los participantes recibieron una encuesta que evaluaba su compromiso afectivo con la institución donde habían recibido servicios de salud y su intención conductual. Al contestar la encuesta los individuos debían escribir el nombre de la última institución de salud que habían visitado como pacientes y pensar en este evento al contestar las preguntas. Se utilizó una escala likert de cinco puntos donde uno significaba totalmente en desacuerdo y cinco significaba totalmente de acuerdo.

La escala de compromiso afectivo contiene tres ítems con base en la escala diseñada por Allen y Mayer (1990), adaptada al español por Frutos, Ruiz y San Martín (1998). La intención conductual, que en este caso se refiere a la intención de usar de nuevo los servicios de una institución, se evaluó a través de tres ítems adaptados al español de Maxham y Netemeyer (2002) por Vázquez Casielles et al. (2007). Finalmente, el compromiso de continuidad se operacionaliza través de la posibilidad de elegir el servicio de salud, la cual fue evaluada al pedir al individuo señalar "si" o "no" a la pregunta: cerca a usted hay muchas instituciones entre las que usted puede escoger. A esta pregunta, el $49 \%$ de 
la muestra contestó afirmativamente (respuesta codificada como uno) y 51\% contestó no tener la posibilidad de elegir (codificada como cero).

\subsection{Resultados}

El análisis de resultados se realiza utilizando el paquete estadístico Amos 4.0 para el análisis confirmatorio y la diferencia de grupos y SPSS versión 23 para el modelo de regresión.

\subsubsection{ANÁLISIS CONFIRMATORIO}

Se verifican la existencia de las variables latentes propuestas para el modelo: compromiso afectivo e intención conductual. Teniendo en cuenta los valores de referencia, los estadísticos observados muestran que existe un buen ajuste de los datos (Hooper, Coughlan y Mullen, 2008). Adicionalmente, para cada escala la carga estandarizada de cada indicador, el índice de fiabilidad $(\rho f)$ y la varianza promedio extraída (AVE) tienen índices superiores al valor de referencia (tabla 1). Así mismo, el Cronbach alfa muestra una alta correlación entre los ítems de cada constructo (Gliem y Gliem, 2003).

\section{Tabla 1}

Propiedades de las escalas e ítems

\begin{tabular}{lc}
\hline Compromiso afectivo: AVE $=0,56 ; \rho f=0,75 ;$ Cronbach $\alpha=0,82$ & $\lambda$ \\
\hline $\begin{array}{l}\text { Disfruto hablando de esta organización con otras personas } \\
\text { No me siento emocionalmente vinculado a esta }\end{array}$ & 0,85 \\
$\begin{array}{l}\text { organización* } \\
\text { Esta institución tiene un significado personal importante } \\
\text { para mí }\end{array}$ & 0,75 \\
\hline $\begin{array}{l}\text { Intención conductual: AVE }=0,70 ; \rho f=0,75 ; \text { Cronbach } \alpha=0,88 \\
\text { Me sentiré feliz si puedo regresar a esta organización y no a }\end{array}$ & 0,85 \\
\hline $\begin{array}{l}\text { otra si tengo que volver a utilizar este tipo de servicios } \\
\text { Mi intención es seguir solicitando los servicios de esta }\end{array}$ & 0,84 \\
$\begin{array}{l}\text { Sinstitución } \\
\text { Si puedo elegir, elegiría de nuevo esta institución }\end{array}$ & 0,82 \\
\hline
\end{tabular}

Índices de ajuste: $X^{2}=16,23$, d.f. $=7, \mathrm{p}=0,02 ; \mathrm{GFI}=0,99(>0,90) ; \mathrm{NFI}=0,99(>0,90) ; \mathrm{TLI}=0,98(>0,95) ; \mathrm{CFI}=0,99$ $(>0,95)$; RMSEA $=0,05(<0,06)$.

Notas: $\lambda$ : carga factorial extraída. AVE: varianza promedio extraída $(>0,5), \rho f$ : índice de fiabilidad compuesta $(>0,6)$. * Los valores se invierten para el análisis. Valores de referencia para ajuste del modelo en paréntesis junto a cada valor estimado.

Fuente: elaboración propia. 


\subsubsection{Modelo de ecuaciones estructurales}

Los resultados de la regresión se presentan con un estilo jerárquico para hacer una mejor descripción de cómo las variables de control no contribuyen al modelo y, lo más importante, la influencia del compromiso de continuidad a través de la posibilidad de elección que tiene el consumidor. El modelo 1 se resume en la ecuación 1. Los resultados en la tabla 2 confirma la hipótesis 1 , validando la relación entre el compromiso afectivo y la intención conductual $(Y)$ del consumidor $(\beta=0,66 ; \beta<0,01)$. Se observa que la intención de uso de un consumidor de servicios de salud aumenta en la medida en que se hace más fuerte su compromiso afectivo hacia la institución $(X)$. Así mismo, la variable dummy que representa la capacidad de elección $(W)$ que percibe el consumidor permite predecir su intención de uso $(\beta=0,08 ; \beta<0,05)$. La intención de uso es mayor para quienes consideran que tienen la capacidad de elegir entre diferentes instituciones de salud en comparación con quienes no tienen esta posibilidad. Las variables de control, género $(G)$ y edad $(E)$, no influyen sobre la intención de uso del consumidor $(p>0,1)$. Finalmente, desde este primer modelo se descartan problemas de multicolinearidad con valores entre 1,01 y 1,03 para el factor de variancia inflacional y valores entre 0,97 y 0,99 para el test de tolerancia.

$Y=a_{0}+a_{1} X+a_{2} W+a_{3} E+a_{4} G+r$

El segundo modelo en la tabla 2, sintetizado en la ecuación 1, permite evaluar en qué medida la relación entre el compromiso afectivo y la intención de uso de una institución de salud se ve afectada por la posibilidad de elección que tiene un consumidor. El efecto moderador representa la interacción entre el compromiso afectivo y la posibilidad de elección (XW) (Preacher, Rucker y Hayes, 2007).

$Y=a_{0}+a_{1} X+a_{2} W+a_{3} X W+r$

Tabla 2

Intención conductual al elegir institución de salud

\begin{tabular}{llll}
\hline \multicolumn{1}{c}{ Variables } & \multicolumn{2}{c}{ Modelo 1} & \multicolumn{2}{c}{ Modelo 2} \\
\hline Compromiso afectivo & $0,66 * * *$ & $0,67 \quad * * *$ \\
Elige & $0,08 \quad * *$ & $0,08 \quad * *$ \\
Género & $-0,03$ & & \\
Edad & 0,00 & & \\
Compromiso afectivo $x$ Elige & & & $-0,02$
\end{tabular}




\begin{tabular}{lccc}
\hline & Variables & Modelo 1 & Modelo 2 \\
\hline $\mathrm{R}^{2}$ Ajustado & 0,46 & 0,46 & \\
$\mathrm{~F}$ & 98,90 & $* * *$ & $131,69 \quad * * *$ \\
\hline
\end{tabular}

Nota: ${ }^{* *} \mathrm{p}<0,05 ;{ }^{* * *} \mathrm{p}<0,01$

Fuente: elaboración propia.

Finalmente, a través de la moderación multigrupo (Gaskin, 2012), se confirma que en ambos grupos, quienes tienen la posibilidad de elegir $(\beta=0,68 ; p<0,01)$ y quienes no la tienen $(\beta=0,70 ; p<0,01)$, existe una relación entre compromiso afectivo e intención conductual. Sin embargo, no existe una diferencia significativa entre los dos grupos con respecto a dicha relación $(Z=0,34 ; p>0,1)$, de manera que no se puede comprobar la interacción y por lo tanto los resultados no soportan la hipótesis 2. En términos de Baron y Kenny (1986), el moderador no tiene la capacidad de cambiar la fuerza o la dirección de la relación entre la variable independiente y la variable dependiente de la ecuación.

\subsection{Discusión}

Este primer estudio muestra como el compromiso afectivo se traduce en una actitud positiva en términos de intención de uso, ratificando los resultados de estudios previos (Evanschitzky et al., 2011; Fullerton, 2005; Gustafsson et al., 2005; Huang et al., 2007; Jones et al., 2010; Omar, Wel, Musa y Nazri, 2010; Wetzels et al., 1998). Adicionalmente, se puede observar que la posibilidad que tiene un consumidor de elegir una institución aumenta su intención de utilizar sus servicios en el futuro. Es decir, hay un efecto positivo al no haber compromiso de continuidad. Sin embargo, esta posibilidad de elección no modera la relación entre el compromiso afectivo y la intención conductual del consumidor.

De esta manera, la limitación que tiene el consumidor a elegir entre un número restringido de instituciones de salud parece no ser una razón de peso para afectar la relación entre el compromiso afectivo del consumidor y su intención de usar la institución. Una explicación para este resultado es que el consumidor no enfrenta la condición de no recibir atención en ninguna institución. La limitación del individuo es la posibilidad de elegir entre pocas o muchas instituciones, esta restricción puede no ser necesariamente un factor negativo para un consumidor. El compromiso de continuidad puede ser un factor positivo si restringir el cambio significa la estabilidad en el prestador del servicio. Incluso, si el usuario de un servicio de salud específico no está familiarizado con dicho servicio el usuario preferirá elegir entre un número limitado de opciones. Por el contrario, en el caso de ser un servicio relativamente común, el usuario preferirá un mayor número de 
alternativas (Bettman et al., 1998; Chernev, 2011; Chernev et al., 2003). Consecuentemente, limitar las opciones no implica negar la posibilidad de recibir un servicio y, por tanto, la posibilidad de elección no tiene un efecto sobre la relación objeto de estudio.

Adicionalmente, los servicios de salud no dependen únicamente de una institución particular sino del seguro de salud de la persona. El cambio o la imposibilidad de cambiar entre una y otra institución no afecta el costo que tiene el servicio para el individuo. Retomando la teoría del compromiso del consumidor, este puede asumir un compromiso de continuidad con base en los costos que anticipa en caso de cambiar de institución (Jones et al., 2010). Así, el siguiente estudio propone evaluar la relación entre el compromiso afectivo y la intención de continuar con una institución para el caso de la educación, caso en el que un cambio implicaría costos importantes para el individuo.

\section{El costo del cambio: continuidad en la formación superior}

Un consumidor puede sentirse obligado a continuar su vínculo con una organización al pensar en los costos que significa terminar dicha relación. Esta es la segunda forma en la que se define el compromiso de continuidad (Meyer et al., 2002). El costo asociado al cambio también se conoce como compromiso calculado (Gustafsson, Johnson y Roos, 2005). Este estudio utiliza la idea del compromiso de continuidad, para evaluar la dependencia de un consumidor hacia una organización al pensar que un cambio le traería más costos que beneficios. En este caso, el compromiso de continuidad es la percepción de pérdidas y ganancias que hace un consumidor en el momento de pensar en no continuar demandando los productos o servicios de una organización (Hur et al., 2010).

Algunas estrategias de mercadeo relacional así como las estrategias de precio y publicidad se asocian a costos de cambio (Polo y Sesé, 2009). El precio es un factor que influye directamente en el costo cuando este es menor que el de la competencia ya sea por una oferta especial o como estrategia de marca. La publicidad se traduce en mayores costos cuando permite que el consumidor aprenda acerca del producto y un cambio de marca significará aprender de nuevo acerca del producto. Por otro lado, el gasto en publicidad puede traducirse al consumidor a través del precio.

Este estudio propone una evaluación más objetiva para determinar que un cambio será realmente más costoso: el hecho de tener o no una beca. El estudio se realiza bajo el marco de una institución de educación superior, contexto en el que la relación entre el usuario y la organización se lleva a cabo por un tiempo prolongado y la mayor retribución por el 
servicio recibido se da en el largo plazo. El hecho de tener una beca es una limitación para el consumidor ya que dicho beneficio está supeditado a continuidad en una universidad. El estudiante no está obligado a continuar con la universidad, pero un cambio significa la pérdida total o parcial del beneficio. Mantener la relación con la institución tiene sentido cuando las ganancias son mayores a las pérdidas de su terminación. Las organizaciones pueden crear estrategias para retener a sus consumidores, pero este objetivo se verá negativamente afectado por el costo asociado a la continuidad de la relación (Fullerton, 2003; Polo y Sesé, 2009).

El compromiso afectivo y la continuidad con una organización dan cuenta de la lealtad del consumidor (Fullerton, 2003, 2005). Sin embargo, la relación entre el compromiso afectivo y la intención de continuar como consumidor se puede fortalecer en el caso en que el consumidor tiene la opción de compararla con otras (Chernev et al., 2003). Por el contrario, la relación entre el compromiso afectivo y la intención de continuar la relación con la institución se verá afectada negativamente si el individuo se siente restringido al cambio (figura 2).

Hipótesis 3: La relación entre el compromiso afectivo de un consumidor y su intención conductual hacia una institución es más fuerte cuando no hay un costo asociado al cambio que cuando existe este costo.

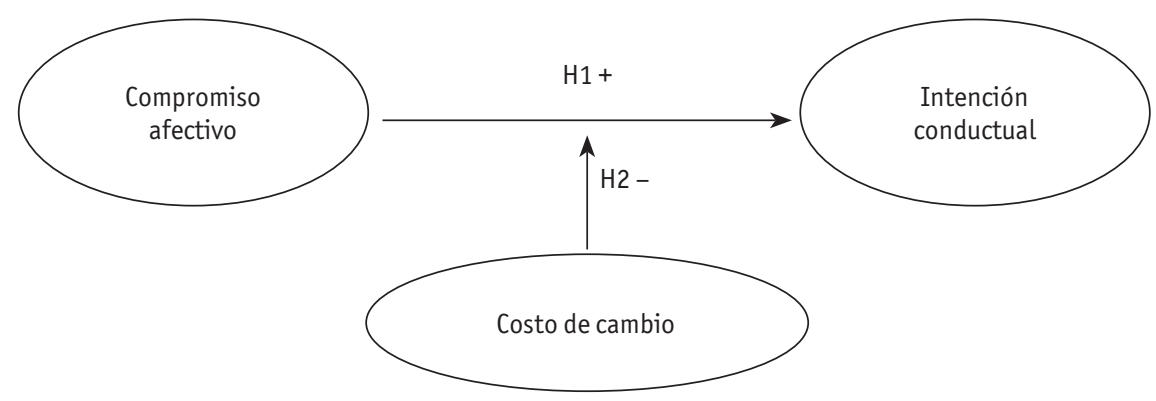

Figura 2. Compromiso de continuidad: el costo del cambio

Fuente: elaboración propia.

\subsection{Estudio 2}

Este estudio evaluó la percepción de estudiantes hacia una institución de educación superior a la cual están vinculados. El reto de este estudio fue proponer una variable que 
permitiera evaluar el compromiso de continuidad no como una percepción del consumidor sino como una variable real. Con este propósito, el estudio se llevó a cabo entre estudiantes de una universidad y se eligió el hecho de tener una beca educativa como variable que condiciona el compromiso de continuidad del individuo. Esta variable supone que el cambio de universidad será más costoso para quienes cuentan con beca en comparación con quienes no reciben este beneficio. La variable fue codificada como uno en el caso de tener beca (costo del cambio) y cero en el caso contrario.

Se contó con 2860 encuestas válidas. Se desecharon 18 encuestas que no fueron diligenciadas correctamente. La muestra estuvo conformada por un $46 \%$ de hombres y $54 \%$ de mujeres con una edad promedio de 20,5 años (desv.st. 2,1). De los encuestados, el $34 \%$ eran estudiantes becados y el $66 \%$ no lo era.

Adicionalmente, de los encuestados el 9,4\% ha cursado un semestre, el $42,7 \%$ ha cursado entre dos y cinco semestres, el $39 \%$ ha cursado entre seis y nueve semestres y el $8,8 \%$ ha cursado diez semestres o más. Se incluyeron estudiantes de todas las carreras de la universidad: Administración 13,9\%, Contabilidad 3,8\%, Economía 18,5\%, Mercadeo 12,2\%, Diseño 11\%, Sistemas 3,5\%, Ingeniería industrial 10,6\%, Telemática 2,5\%, Antropología 1,3\%, Ciencias políticas 2,7\%, Derecho 5,1\%, Psicología 2,7\%, Sociología 0,9\%, Biología 1,3\%, Química 6,3\% y Medicina 3,6\%.

Los estudiantes fueron invitados a participar en un estudio con el objetivo de evaluar su percepción hacia la universidad. La encuesta incluyó tres ítems para evaluar el compromiso afectivo (Allen y Meyer, 1990). Estos ítems fueron adaptados del estudio anterior para el caso de una institución de educación superior.

Así mismo, los ítems que evaluaron la variable dependiente, intención conductual, fueron paralelos a los del primer estudio siendo adaptados al entorno educativo. Ambos constructos se evaluaron utilizando una escala likert de cinco puntos con un rango entre totalmente de acuerdo y totalmente en desacuerdo.

\subsection{Resultados}

Análisis confirmatorio. Se verifica la existencia de las variables latentes propuestas para el nuevo modelo (tabla 3). Los estadísticos confirman que existe un buen ajuste y fiabilidad de los datos teniendo en cuenta los valores de referencia (Hooper et al., 2008). 


\section{Tabla 3}

Propiedades de las escalas e ítems

\begin{tabular}{lc}
\hline Compromiso afectivo: AVE $=0,66 ; \rho f=0,67 ;$ Cronbach $\alpha=0,88$ & $\lambda$ \\
\hline $\begin{array}{l}\text { Disfruto hablando de la Universidad con otras personas } \\
\text { Quiero mucho a mi Universidad }\end{array}$ & 0,84 \\
$\begin{array}{l}\text { La Universidad tiene un significado importante para mí } \\
\text { Intención conductual: AVE }=0,69 ; \rho f=0,75 ; \text { Cronbach } \alpha=0,79\end{array}$ & $\lambda$ \\
\hline $\begin{array}{l}\text { Si tuviera que volver a elegir, seleccionaría de nuevo esta } \\
\text { universidad para estudiar }\end{array}$ & 0,91 \\
$\begin{array}{l}\text { Al terminar mi carrera regresaré a esta universidad para } \\
\text { realizar mis estudios de postgrado }\end{array}$ & 0,73 \\
$\begin{array}{l}\text { Aunque existan otras universidades con la misma carrera, } \\
\text { deseo continuar estudiando en esta }\end{array}$ & 0,85 \\
\hline
\end{tabular}

Índices de ajuste: $X^{2}=13,97$, d.f. $=3, \mathrm{p}=0,00 ; \mathrm{GFI}=0,99(>0,90) ; \mathrm{NFI}=0,99(>0,90) ; \mathrm{TLI}=0,99(>0,95)$; $\mathrm{CFI}=0,99(>0,95)$; RMSEA=0,04 $(<0,06)$.

Notas: $\lambda$ : carga factorial extraída. AVE: varianza promedio extraída $(>0,6) . \rho f$ : índice de fiabilidad compuesta $(>0,6)$. * Ítems eliminados durante el proceso de purificación. Valores de referencia para ajuste del modelo en paréntesis junto a cada valor estimado.

Fuente: elaboración propia.

Modelo de ecuaciones estructurales. En la tabla 4 se presentan los resultados del modelo de regresión (ecuación 1). La hipótesis 1 se confirma a partir del modelo 1 en la que un mayor compromiso afectivo $(X)$ por una institución educativa significa una mayor intención de permanecer $(Y)$ con la institución $(\beta=0,67 ; \beta<0,01)$. La variable dummy “beca” (W) no permite afirmar que de manera directa una restricción asociada a un costo a favor del estudiante influya directamente en su intención conductual $(\beta=0,01 ; p>0,1)$. Tampoco se observa que la edad $(E)$ o el género $(G)$ tengan relación con la intención conductual de un individuo hacia la institución $(p>0,1)$. Al no observar un efecto en las variables de control, estas se descartan del modelo. Adicionalmente, no se encuentran problemas de multicolinearidad entre las variables independientes encontrando valores para el factor de inflación de la varianza entre 1,01 y 1,03 y valores de tolerancia entre 0,97 y 0,99.

El segundo modelo evalúa el cambio en la relación entre el compromiso afectivo y la intención conductual del individuo dependiendo de una tercera variable, beca. Con este objetivo se incluye la interacción (XW) entre el hecho de tener beca y el compromiso afectivo del individuo (ecuación 2).

Después de tener en cuenta el compromiso afectivo $(\beta=0,7 ; p<0,01)$ y el hecho de tener o no una beca $(\beta=0,01 ; p>0,1)$, se observa que la interacción entre dichas variables es 
significativa, confirmando la hipótesis 3. Así, el hecho de tener beca implica que la relación ente el compromiso afectivo y la intención de continuar en la institución es más débil en comparación con las personas que no tienen beca $(\beta=-0,04 ; p<0,01)$. El efecto moderador se confirma al evaluar directamente la diferencia entre grupos en la relación compromiso afectivo - intención conductual; esto obedece a una moderación multigrupo (Gaskin, 2012). Para el grupo de estudiantes que tiene beca, un mayor compromiso afectivo aumenta su intención conductual hacia la institución $(\beta=0,61 ; p<0,01)$. La misma relación es cierta para quienes no tiene beca $(\beta=0,69 ; p<0,01)$. Sin embargo, existe una diferencia significativa entre ambos grupos, siendo más fuerte la relación entre compromiso afectivo e intención conductual para quienes no tienen beca $(Z=2,71 ; p<0,01)$, comprobando la interacción.

\section{Tabla 4}

Intención conductual cuando el cambio tiene un costo (beca educativa)

\begin{tabular}{lccc}
\hline \multicolumn{1}{c}{ Variables } & \multicolumn{1}{c}{ Modelo 1} & \multicolumn{2}{c}{ Modelo 2} \\
\hline Compromiso afectivo & $0,66 \quad * * *$ & $0,69 \quad * * *$ \\
Beca (costo del cambio) & 0,01 & 0,01 \\
Género & $-0,01$ & & \\
Edad & $-0,03$ & & \\
Compromiso afectivo x Beca & & & $-0,04 \quad * * *$ \\
$\mathrm{R}^{2}$ Ajustado & 0,44 & 0,44 & \\
$\mathrm{~F}$ & 552,58 & $* * *$ & $757,08 \quad * * *$ \\
\hline
\end{tabular}

Nota: ** $\mathrm{p}<0,05 ;{ }^{* * *} \mathrm{p}<0,01$

Fuente: elaboración propia.

\subsection{Discusión}

La interacción entre el compromiso afectivo y la condición que retiene al consumidor a través de un costo/beneficio permite entender mejor la relación entre su compromiso afectivo e intención conductual hacia la organización. A pesar de que el vínculo con una institución educativa es una relación de largo plazo, no es la permanencia de la relación entre el consumidor y la organización a través del tiempo lo que genera el vínculo. Como se afirma desde la teoría es la emoción que el consumidor experimenta por la organización lo que le lleva a conservar el vínculo afectivo y permanecer con la organización al demandar sus servicios. En el caso en que el vínculo emocional se asocie a una obligación, a pesar de que esta sea un beneficio, el consumidor tendrá una menor inclinación a continuar vinculado a la institución. 
Este resultado es coherente con la idea de que es el compromiso afectivo, y no el compromiso de continuidad, el que genera lealtad en el consumidor (Fullerton, 2003). Este resultado permite entender como en muchos casos las organizaciones crean estrategias de fidelización que atan al consumidor a la organización con el atractivo de un beneficio que se podrá obtener en el largo plazo (Zhang y Breugelmans, 2012). En efecto, el consumidor continúa su relación con la organización porque hay una limitación que restringe el cambio al crear un costo; sin embargo esto puede estar en detrimento del compromiso afectivo que el consumidor siente por la organización. Incluso, en otros estudios, después de tener en cuenta el nivel de compromiso del consumidor, no se observa una relación directa entre su satisfacción y lealtad hacia una organización (0mar, Alam, Aziz y Nazri, 2011; 0mar et al., 2010). Es decir, la percepción de obligación parece romper el vínculo que consistentemente se observa entre la satisfacción y la recompra.

En otros casos se observa que aquellos individuos con un mayor compromiso hacia la institución son más críticos hacia ella, muestran mayor intención de quejarse y no se recuperan fácilmente de un mal servicio (Evanschitzky et al., 2011). Esta actitud crítica y el saber que hay un alto costo asociado al cambio, parece tener como consecuencia una relación más débil entre el compromiso afectivo y la intención conductual, en comparación con el hecho de que no exista la obligación al vínculo con la institución.

\section{Discusión general}

Los dos estudios realizados evalúan la fidelidad de un consumidor a continuar con una organización utilizando dos operacionalizaciones diferentes, lo que permite fortalecer la validez del constructo. El primer estudio entiende la obligación como la posibilidad de elección y el segundo como el costo asociado a no continuar con una institución. Ambas perspectivas hacen parte de la definición del compromiso de continuidad, pero la literatura muestra que en ambos casos el constructo se ha operacionalizado según el nivel de acuerdo que tiene el consumidor con estos aspectos (Fullerton, 2003, 2005). Por el contario, este estudio realiza para ambos aspectos una medición independiente y objetiva utilizando hechos concretos: el tener o no la posibilidad de elegir y el tener o no una beca educativa.

En el primer caso, el efecto moderador de la posibilidad de elegir un servicio de salud no fue soportado, pero sí se observa una relación directa entre el compromiso de continuidad (posibilidad de elegir) y la intención conductual. Es importante notar que la posibilidad de elegir entre los diferentes oferentes del mercado hace parte de la definición del 
compromiso de continuidad pero no es la forma como habitualmente se operacionaliza este constructo. Previamente, su operacionalización se ha inclinado hacia el costo del cambio o la facilidad de cambio por razones personales, no se había definido en términos de restricciones del mercado y amplitud de la oferta (Fullerton, 2003, 2005). Así, esta extensión y adaptación de la operacionalización del concepto de compromiso de continuidad permite fortalecer la validez del constructo.

El segundo estudio evalúa el tener una beca educativa como un hecho que obliga a continuar el vínculo con la institución ya que el cambio sería costoso para el individuo. Esta segunda forma de compromiso de continuidad mostró un efecto negativo sobre la relación entre el compromiso afectivo y la intención conductual. A pesar de que la literatura se inclina más hacia este concepto al operacionalizar el constructo compromiso de continuidad, este estudio muestra una operacionalización complementaria, más sencilla y objetiva, fortaleciendo también la validez del constructo.

En síntesis, este set de estudios confirma la relación positiva entre el compromiso afectivo y las intenciones conductuales de un consumidor hacia una organización. Los resultados muestran que las dos formas de compromiso de continuidad afectan de manera diferente la intención conductual del consumidor hacia la organización.

\section{Limitaciones y recomendaciones para futuros estudios}

En los dos estudios realizados, el consumidor establece con la organización una relación que es diferente en el tiempo. En el primer estudio, el caso de servicios de salud, la relación de consumo puede ocurrir en un periodo de tiempo relativamente corto. Mientras tanto, en el segundo estudio, el caso de la educación superior, la relación de consumo ocurre en un periodo de cinco años aproximadamente. Así, aunque no es posible hacer una comparación directa entre los dos casos, y por tanto no es una comparación concluyente, es posible pensar que se establece un vínculo emocional más fuerte en relaciones consumidor-organización que se piensan en el largo plazo. El compromiso de continuidad para el caso de una institución educativa, no solo está directamente relacionada al costo evaluado a través de la beca, es posible que el efecto moderador de la obligación a continuar con una organización ocurra también gracias al tiempo en el que se establece el vínculo entre el consumidor y la organización.

Estudios previos demuestran que la dimensión temporal es frágil y se altera fácilmente en el momento en que el consumidor debe hacer una elección o señalar sus preferencias en 
el tiempo (Eberty Prelec, 2007). Consistentemente, no es igual la percepción del tiempo de compromiso para un servicio que se establece en el corto plazo a uno que se establece en el largo plazo. Asociado a esta comparación, este estudio no evalúa el compromiso afectivo para el caso de marcas o productos que se utilicen y desechen rápidamente. Estas son situaciones en las que el consumidor establece un vínculo de corto plazo. Futuros estudios pueden evaluar en qué medida una condición de obligación hacia una marca influye en la relación entre el compromiso afectivo y la intención conductual del consumidor para el caso de marcas o productos de consumo masivo.

Por otro lado, los resultados del segundo estudio señalan que el hecho de tener una beca conlleva a una relación menos fuerte entre el compromiso afectivo y la intención conductual en comparación con quienes no tienen este beneficio. Futuros estudios deben confirmar la experiencia emocional de los consumidores que reciben beneficios adicionales. Es difícil pensar que estudiantes que reciben un beneficio económico adicional no se encuentren agradecidos con la institución. Este resultado merece validarse a través de otras categorías de productos.

Los resultados sugieren que el compromiso de continuidad que se crea a través de una limitación en comparación con el compromiso de continuidad que se crea por un beneficio adicional no son estrategias equivalentes. Es decir, no interactúan de la misma manera con el compromiso afectivo del consumidor. En este caso, sería necesario entender si es que las obligaciones que se formulan de manera positiva no tienen el mismo efecto que aquellas que se formulan de manera negativa. Adicionalmente, estas dos formas de compromiso de continuidad que se utilizan en el estudio son independientes entre sí. Aunque la teoría indica que son parte del mismo constructo, este estudio no evalúa su consistencia de manera estadística. Futuros estudios pueden confirmar la consistencia de los resultados entre ambas perspectivas, la posibilidad de elegir y el costo del cambio, como para ser ambos parte del compromiso de continuidad.

\section{Implicaciones prácticas}

Pensando en gerentes, administradores y ejecutivos de mercadeo, se deben entender las relaciones con el consumidor como una ventaja competitiva. El valor diferencial de una marca no se construye a través de la obligación sino de la decisión personal del consumidor de apreciar la marca como única. De esta manera, es necesario entender el efecto de las estrategias de retención y cuáles son aquellas que específicamente generan lealtad en 
el consumidor (Fandos, Sánchez, Moliner y Estrada, 2011) o, por el contrario, generan la percepción de obligación afectando negativamente la lealtad.

Las estrategias de mercadeo que dan al consumidor la posibilidad de elección tienen una influencia directa y positiva sobre la intención conductual del consumidor hacia la organización. Por el contrario, las estrategias que ofrecen un beneficio en términos de costos no tienen una influencia directa sobre la intención conductual del consumidor; este tipo de estrategias deben considerar su efecto a través del compromiso afectivo. El beneficio percibido a través del menor costo puede potencializar el compromiso afectivo hacia la organización y asegurar la retención del consumidor (Hur et al., 2010).

Para una marca y organización es importante entender a sus consumidores, diferenciando aquel que establece una relación de acuerdo con su vínculo afectivo de aquel para el cual la relación además tiene en cuenta la conveniencia en términos costo/beneficio. Adicionalmente, la evaluación de conveniencia por el análisis costo/beneficio que hace un consumidor es una evaluación que permite a la organización entender realmente su ventaja competitiva (Gustafsson et al., 2005). Es decir, un consumidor que prefiere una marca, después de evaluar sus beneficios dados los costos relativos, es un consumidor realmente leal a la marca. La influencia que tiene la conciencia del consumidor sobre el costo/beneficio en su decisión de continuar con una marca, refuerza la recomendación de estudios de mercadeo relacional señalando que aquellos consumidores más vinculados a la marca son los que más atención merecen. Así, el beneficio asociado al costo no debe ser percibido por el consumidor como una obligación; de esta forma, la estrategia jugará en contra de la lealtad.

\section{Referencias}

Allen, N. J., and Meyer, J. P. (1990). The measurement and antecedents of affective, continuance and normative commitment to the organization. Journal of Occupational Psychology, 63, 1-18.

Baron, R. M., and Kenny, D. A. (1986). The moderator-mediator variable distinction in social psychological research: Conceptual, strategic, and statistical considerations. Journal of Personality and Social Psychology, 51 (6), 1173-1182.

Bettman, J. R., Luce, M. F., and Payne, J. W. (1998). Constructive consumer choice processes. Journal of Consumer Research, 25 (3), 187-217.

Bowlby, J. (1977). The making and breaking of affectional bonds. The British Journal of Psychiatry $130,421-431$. 
Chernev, A. (2011). When more is less and less is more: The psychology of managing product assortments. Marketing Intelligence Review, 3 (1), 8-15.

Chernev, A., Mick, D. G., and Johnson, M. D. (2003). When more is less and less is more: The role of ideal point availability and assortment in consumer choice. Journal of Consumer Research, 30 (2), 170-183.

Ebert, J. E. J., and Prelec, D. (2007). The fragility of time: Time-insensitivity and valuation of the near and far future. Management Science, 53 (9), 1423-1438.

Evanschitzky, H., Brock, C., and Blut, M. (2011). Will you tolerate this? The impact of affective commitment on complaint intention and postrecovery behavior. Journal of Service Research, 14 (4), 410-425.

Fandos, J. C., Sánchez, J., Moliner, M. Á., y Estrada, M. (2011). La lealtad del consumidor en el sector financiero. Innovar. Revista de Ciencias Administrativas y Sociales, 21 (39), 39-52.

Frutos, B. d., Ruiz, M. A., y San Martín, R. (1998). Análisis factorial confirmatorio de las dimensiones del compromiso con la organización. Psicológica, 19, 345-366.

Fullerton, G. (2003). When does commitment lead to loyalty? Journal of Service Research, 5 (4), 333-344.

Fullerton, G. (2005). The impact of brand commitment on loyalty to retail service brands. Canadian Journal of Administrative Sciences, 22 (2), 97-110.

Gaskin J. Stats Tools Package: Group Differences. Gaskination's StatWiki (2012). Accessed from http://statwiki.kolobkreations.com/index.php?title=Main_Page

Gautam, V., and Kumar, M. (2011). An empirical investigation of factors determining the consumers' choice of mobile service providers. Journal of Business Research, 3 (4), 3-17.

Gliem, J., and Gliem, R. (2003). Calculating, interpreting, and reporting Cronbach's alpha reliability coefficient for Likert-type scales. Paper presented at the Midwest Research to Practice Conference in Adult, Continuing, and Community Education. Accessed from http://www. alumni-osu.org/midwest/proceeding.html

Gustafsson, A., Johnson, M. D., and Roos, I. (2005). The effects of customer satisfaction, relationship commitment dimensions, and triggers on customer retention. Journal of Marketing, 69 (4), 210-218.

Hooper, D., Coughlan, J., and Mullen, M. (2008). Structural equation modelling : guidelines for determining model fit.Dublin Institute of Technology ARROW @ DIT, 6 (1),53-60.

Huang, L.-T., Cheng, T.-C., and Farn, C.-K. (2007). The mediating effect of commitment on customer loyalty towards e-brokerages: An enhanced investment model. Total Quality Management \& Business Excellence, 18 (7), 751-770.

Hur, W.-M., Park, J., and Kim, M. (2010). The role of commitment on the customer benefits-loyalty relationship in mobile service industry. Service Industries Journal, 30 (14), 2293-2309. 
Jones, T., Fox, G. L., Taylor, S. F., and Fabrigar, L. R. (2010). Service customer commitment and response. Journal of Services Marketing, 24 (1), 16-28.

Maxham, J. G., and Netemeyer, R. G. (2002). Modeling customer perceptions of complaint handling over time: the effects of perceived justice on satisfaction and intent. Journal of Retailing, 78 (4), 239-252.

Meyer, J. P., Stanley, D. J., Herscovitch, L., and Topolnytsky, L. (2002). Affective, continuance, and normative commitment to the organization: A meta-analysis of antecedents, correlates, and consequences. Journal of Vocational Behavior, 61 (1), 20-52.

Morgan, R. M., and Hunt, S. D. (1994). The commitment-trust theory of relationship marketing. The Journal of Marketing, 58 (3), 20-38.

Omar, N. A., Alam, S. S., Aziz, N. A., and Nazri, M. A. (2011). Retail Loyalty Programs in Malasia: the relationship of equity, value, satisfaction, trust, and loyalty among cardholders. Journal of Business Economics \& Management, 12 (2), 332-352.

Omar, N. A., Wel, C. A. C., Musa, R., and Nazri, M. A. (2010). Program benefits, satisfaction and loyalty in retail loyalty program: Exploring the roles of program trust and program commitment. IUP Journal of Marketing Management, 9 (4), 6-28.

Palmatier, R. W., Jarvis, C. B., Bechkoff, J. R., and Kardes, F. R. (2009). The role of customer gratitude in relationship marketing. Journal of Marketing , 73 (Sept.), 1-18.

Polo, Y., and Sesé, F. J. (2009). How to make switching costly: The role of marketing and relationship characteristics. Journal of Service Research, 12 (2), 119-137.

Preacher, K. J., Rucker, D. D., and Hayes, A. F. (2007). Addressing moderated mediation hypotheses: Theory, methods, and prescriptions. Multivariate Behavioral Research, 42, 185-227.

Sundie, J. M., Gelb, B. D., and Bush, D. (2008). Economic reality versus consumer perceptions of monopoly. Journal of Public Policy \& Marketing, 27 (2), 178-181.

Vázquez-Casielles, R., Suárez-Alvarez, L., Díaz-Martín, A. M., y Del Río-Lanza, A. B. (2007). Justicia percibida y satisfacción del cliente con la solución del problema como determinantes de la lealtad en contextos de fallo de servicio. Documento presentado en el XX Congreso anual de AEDEM. Recuperado de http://dialnet.unirioja.es/servlet/articulo? codigo $=2486883$

Wetzels, M., de Ruyter, K., and van Birgelen, M. (1998). Marketing service relationships: the role of commitment. The Journal of Business \& Industrial Marketing, 13 (4/5), 406.

Zhang, J., and Breugelmans, E. (2012). The impact of an item-based loyalty program on consumer purchase behavior. Journal of Marketing Research, 49 (1), 50-65.

Zhao, Y., Zhao, Y., and Helsen, K. (2011). Consumer learning in a turbulent market environment: Modeling consumer choice dynamics after a product-harm crisis. Journal of Marketing Research, 48 (2), 255-267. 
